\title{
A Single Amino Acid at Position 158 in Hemagglutinin Affects the Antigenic Property of Eurasian Avian-like H1N1 Swine Influenza Viruses
}

Zeng Wang ${ }^{1}$, Yan Chen ${ }^{2}$, Huayuan Chen ${ }^{1}$, Fei Meng ${ }^{2}$, Shiyu Tao ${ }^{2}$, Shujie Ma ${ }^{2}$, Chuanling Qiao $^{2}$, Hualan Chen ${ }^{2}$, and huanliang yang ${ }^{2}$

${ }^{1}$ College of Veterinary Medicine Henan Agricultural University Zhengzhou People's Republic of China

${ }^{2}$ State Key Laboratory of Veterinary Biotechnology Harbin Veterinary Research Institute Chinese Academy of Agricultural Sciences (CAAS) Harbin People's Republic of China

May 26, 2021

\begin{abstract}
Influenza viruses have been posing a great threat to public health and animal industry. The developed vaccines have been widely used to reduce the risk of potential pandemic; however, the ongoing antigenic drift makes influenza virus escape from host immune response and hampers vaccine efficacy. Until now, the genetic basis of antigenic variation remains largely unknown. In this study, we used A/swine/Guangxi/18/2011 (GX/18) and A/swine/Guangdong/104/2013 (GD/104) as models to explore the molecular determinant for antigenic variation of Eurasian avian-like H1N1 (EA H1N1) swine influenza viruses (SIVs), and found that the GD/104 virus exhibited 32 64-fold lower antigenic cross-reactivity with antibodies against GX/18 virus. Therefore, we generated polyclonal antibodies against GX/18 or GD/104 virus and a monoclonal antibody (mAb), named mAb102-95, targeted to the hemagglutinin (HA) protein of GX/18 virus, and found that a single amino acid substitution at position 158 in HA protein substantially altered the antigenicity of virus. The reactivity of GX/18 virus containing G158E mutation with the mAb102-95 decreased 8-fold than that of the parental strain. Contrarily, the reactivity of GD/104 virus bearing E158G mutation with the mAb102-95 increased by 32 times as compared with that of the parental virus. Structural analysis showed that the amino acid mutation from $\mathrm{G}$ to $\mathrm{E}$ was accompanied with the $\mathrm{R}$ group changing from $-\mathrm{H}$ to $-(\mathrm{CH}$ 2) 2 - $\mathrm{COOH}$. The induced steric effect and increased hydrophilicity of HA protein surface jointly contributed to the antigenic drift of EA H1N1 SIVs. Our study provides experimental evidence that G158E mutation in HA protein affects the antigenic property of EA H1N1 SIVs, and widens our horizon on the antigenic drift of influenza virus.
\end{abstract}

\section{A S ingle}

Amino Acid at Position 158 in Hemagglutinin Affects the Antigenic Property of Eurasian Avian-like H1N1 Swine Influenza Viruses

Zeng Wang ${ }^{1,+}$, Yan Chen ${ }^{2,+}$, Huayuan $\mathrm{Chen}^{1}$, Fei Meng ${ }^{2}$, Shiyu Tao ${ }^{2}$, Shujie $\mathrm{Ma}^{2}$, Chuanling Qiao ${ }^{2}$, Hualan Chen $^{2}$, Huanliang Yang ${ }^{2, *}$

${ }^{1}$ College of Veterinary Medicine, Henan Agricultural University, Zhengzhou, People's Republic of China

${ }^{2}$ State Key Laboratory of Veterinary Biotechnology, Harbin Veterinary Research Institute, Chinese Academy of Agricultural Sciences (CAAS), Harbin, People's Republic of China

Running title: G158E mutation in HA affects the antigenicity of EA H1N1 SIVs 


\section{${ }^{*}$ Corresponding author:}

Huanliang Yang,

State Key Laboratory of Veterinary Biotechnology, Harbin Veterinary Research Institute, Chinese Academy of Agricultural Sciences (CAAS), Harbin, People's Republic of China

E-mail: yanghuanliang@caas.cn

+Zeng Wang and Yan Chen contributed equally to this study.

\section{Summary:}

Influenza viruses have been posing a great threat to public health and animal industry. The developed vaccines have been widely used to reduce the risk of potential pandemic; however, the ongoing antigenic drift makes influenza virus escape from host immune response and hampers vaccine efficacy. Until now, the genetic basis of antigenic variation remains largely unknown. In this study, we used A/swine/Guangxi/18/2011 (GX/18) and A/swine/Guangdong/104/2013 (GD/104) as models to explore the molecular determinant for antigenic variation of Eurasian avian-like H1N1 (EA H1N1) swine influenza viruses (SIVs), and found that the GD/104 virus exhibited 32 64-fold lower antigenic cross-reactivity with antibodies against GX/18 virus. Therefore, we generated polyclonal antibodies against GX/18 or GD/104 virus and a monoclonal antibody (mAb), named mAb102-95, targeted to the hemagglutinin (HA) protein of GX/18 virus, and found that a single amino acid substitution at position 158 in HA protein substantially altered the antigenicity of virus. The reactivity of GX/18 virus containing G158E mutation with the mAb102-95 decreased 8-fold than that of the parental strain. Contrarily, the reactivity of GD/104 virus bearing E158G mutation with the mAb102-95 increased by 32 times as compared with that of the parental virus. Structural analysis showed that the amino acid mutation from $\mathrm{G}$ to $\mathrm{E}$ was accompanied with the $\mathrm{R}$ group changing from $-\mathrm{H}$ to $-\left(\mathrm{CH}_{2}\right)_{2}-\mathrm{COOH}$. The induced steric effect and increased hydrophilicity of HA protein surface jointly contributed to the antigenic drift of EA H1N1 SIVs. Our study provides experimental evidence that G158E mutation in HA protein affects the antigenic property of EA H1N1 SIVs, and widens our horizon on the antigenic drift of influenza virus.

Key words: Eurasian avian-like H1N1 SIVs; antigenic drift; hemagglutinin; G158E

\section{Introduction}

Influenza A virus (IAV) is an important respiratory pathogen that continually impacts human public health and the animal industry. The wild waterfowl has been thought to be the natural reservoir for IAV, however, the viruses frequently jump species barriers and infect humans and other mammals, such as pigs, cats, horses, and whales(Zhu et al., 2019a). Swine is of particular significance due to its susceptibility to avian, swine, and human influenza viruses and has been regarded as "gene mixing vessels" to generate virus with pandemic potential(Ma et al., 2008, Ito et al., 1998). Systematic surveillance of swine influenza viruses (SIVs) is essential for early warning and preparedness for the next potential pandemic. In China, pigs are not vaccinated against influenza virus, and distinct lineages of SIVs, such as classical swine H1N1 (CS H1N1), Eurasian avian-like H1N1 (EA H1N1), and triple reassortant H3N2 (TR H3N2), have been co-circulating in pig herds(Zhu et al., 2019b). EA H1N1 SIVs were firstly transmitted from waterfowl to pigs in 1979 in Europe(Pensaert et al., 1981) and gradually became the predominant lineage in China(Yang et al., 2016). Since 2009, the 2009 pandemic (pdm/09) H1N1 in humans quickly transmitted to pigs(Weingartl et al., 2010, Pereda et al., 2010), and genetic reassortants between EA H1N1 SIVs and pdm/09 H1N1 have been frequently reported among pigs(Zhu et al., 2011, Cao et al., 2019). Notably, sporadic human infections of EA H1N1 and other reassortant SIVs highlight the importance of influenza surveillance in pigs and humans(Yang et al., 2012, Zhu et al., 2016b, Xie et al., 2018).

As a multifunctional protein, a single amino acid mutation in hemagglutinin (HA) can largely alter the biological property of virus. For example, G225E mutation in HA has been confirmed to significantly improve the respiratory droplet transmission of EA H1N1 SIVs in guinea pigs(Wang et al., 2017). Neutralizing 
antibodies stimulated by HA protein can protect humans or animals against influenza virus infection. HA protein consists of two polypeptides, HA1 and HA2, and HA1 plays much important roles than HA2 in triggering host immune response(Chi et al., 2005). Accumulation of amino acid mutations in HA1 usually cause antigenic drift, making the vaccine unable to offer effective protection against antigenically mismatched circulating strains. When the prevalent viruses show a greater than 4 -fold difference in hemagglutinin inhibition (HI) assay titer from the vaccine strain, the immunity induced by the vaccine does not prevent the circulation of such viruses in population(Smith et al., 2001). Therefore, identification of the genetic determinants for antigenic variation will be undoubtedly invaluable for vaccine development and prevention of influenza virus outbreaks.

Currently, EA H1N1 SIVs have been frequently recombining with other influenza viruses, such as pdm/09 H1N1 and TR H3N2(He et al., 2018). Importantly, the overwhelming majority of the reassortants preserved the HA and NA genes of EA H1N1 SIVs(Sun et al., 2020), indicating that studying the genetic basis for antigenic drift of EA H1N1 SIVs is essential to evaluate the antigenic properties of SIVs. Our previous study demonstrated that EA H1N1 SIVs formed two distinct antigenic groups, and A/swine/Guangdong/104/2013 (GD/104) virus exhibited 32 64-fold lower antigenic cross-reactivity with antibodies against A/swine/Guangxi/18/2011 (GX/18) virus(Yang et al., 2016). In this study, we used GX/18 and GD/104 as models to explore the underlying mechanism of this difference in antigenicity.

\section{Materials and methods}

\subsection{Ethics statement and facility.}

All animal experiments were carried out in strict accordance with the recommendations in the Guide for the Care and Use of Laboratory Animals of the Ministry of Science and Technology of the People's Republic of China. All studies were conducted in a biosecurity level 2 laboratory approved for such use by the Harbin Veterinary Research Institute (HVRI) of the Chinese Academy of Agricultural Sciences (CAAS). The protocol was approved by the Committee on the Ethics of Animal Experiments of the HVRI of the CAAS.

\subsection{Viruses and cells.}

The EA H1N1 SIVs, A/swine/Guangxi/18/2011 (GX/18) and A/swine/Guangdong/104/2013 (GD/104), were isolated in slaughterhouse during routine surveillance from 2010 to 2013 . HEK293T and Sp2/0 cells were maintained in Dulbecco's modified Eagle's medium and RPMI 1640 medium with $10 \%$ fetal calf serum at $37^{\circ} \mathrm{C}$ in $5 \% \mathrm{CO}_{2}$, respectively.

\subsection{Antisera and monoclonal antibody preparation.}

Antisera from ferrets against GX/18 or GD/104 were obtained from our previous study(Yang et al., 2016), and counterparts from guinea pigs were generated by using six-week-old animals (Vital River Laboratories, Beijing, China) that were serologically negative for circulating seasonal influenza A and B viruses. Two guinea pigs were intranasally inoculated with $10^{6} \mathrm{EID}_{50}$ of each virus in a volume of $300 \mu \mathrm{l}$ (150 $\mu \mathrm{l}$ per nostril), and the antisera were collected 3 weeks after inoculation by euthanizing the animals. After being treated with vibrio choleraereceptor-destroying enzyme (DenkaSeiken) for $18 \mathrm{~h}$ at $37^{\circ} \mathrm{C}$ and heat-inactivated at $56^{\circ} \mathrm{C}$ for $30 \mathrm{~min}$, the antisera were ready for $\mathrm{HI}$ assay. The mAb used in this study was produced according to the standard protocol previously described by $\mathrm{Li}$ et al .(Li et al., 2017). Briefly, BALB/c mice were immunized intraperitoneally with $500 \mu \mathrm{l} \beta$-Propiolactone-inactive GX/18 virus (with $2^{8}$ hemagglutination unit) at 6-, 9-, and 11-weeks old. Three days after the final immunization, the mice were euthanized and splenocytes were collected. The spleen cells were fused with Sp2/0 cells to produce a hybridoma as described by Gefter et al .(Gefter et al., 1977), and cloned in 96-well plate with HAT medium. The positive hybridoma clones were screened through HI assay and then subcloned four times by limiting dilution method. Finally, the cloned hybridoma cells were injected into the peritoneum of the BALB/c mice, and abdominal ascites were collected 7 days later.

\subsection{Antigenic cartography.}


Analysis of antigenic properties was performed using the antigenic cartography methods described previously(Smith et al., 2004). The antigen cartography of the viruses was constructed by using Antigenic Cartography software (http://www.antigenic-cartography.org/).

\subsection{Virus rescue.}

The eight gene segments of GX/18 and GD/104 were inserted into the vRNA-mRNA bidirectional transcription vector $\mathrm{pBD}$ to rescue $\mathrm{rGX} / 18, \mathrm{rGD} / 104$ and the reassortant and mutant viruses as described previously(Ping et al., 2008). Mutations were introduced into HA gene in the background of GX/18 and GD/104 by site-directed mutagenesis (Invitrogen) according to the manufacturer's protocol. Gene-specific primers used in study were shown in Table 1, and all the rescued viruses were completely sequenced to avoid the unwanted mutations.

\subsection{HI assay.}

The HI assay was performed following the standard methods. Briefly, $25 \mu \mathrm{l}$ of 2 -fold serially diluted antisera or mAb were mixed with $25 \mu \mathrm{l}$ of $4 \mathrm{HA}$ units of tested virus. After incubating at room temperature for 30 min, $50 \mu \mathrm{l}$ of $0.5 \%$ chicken red blood cells were added to each well and incubated at room temperature for 40 min. The HI titer was defined as the reciprocal of the highest serum dilution that completely inhibited hemagglutination.

\section{Results}

\subsection{EA H1N1 SIVs form two distinct antigenic groups.}

Preexisting immunity can protect hosts from related influenza viruses, but antigenic drift can decrease the protective efficacy. Yang et al. previously evaluated the antigenic relationship of EA H1N1 SIVs isolated from 2010 to 2013(Yang et al., 2016), and we quantitatively analyzed their cross-reactivity with ferret antisera using the methods described by Smith et al .(Smith et al., 2004). The 10 EA H1N1 SIVs could be classified into two different antigenic groups A and B (Figure 1). Most of them were in group A, while two viruses, A/swine/Guangdong/104/2013 and A/swine/Guangdong/306/2013, belonged to group B. These results demonstrated that the isolated EA H1N1 SIVs form two distinct antigenic groups, but the underlying genetic determinants for the antigenic variation remains unclear.

\subsection{The GD/104 virus exhibits low antigenic cross-reactivity with antibodies against GX/18} virus.

The representative viruses in group A and B, GX/18 and GD/104, were used as models to explore the genetic basis for antigenic drift of EA H1N1 SIVs. To further investigate their antigenic relationship, ferret, and guinea pig antisera against the two viruses and a monoclonal antibody (mAb), named mAb102-95, targeted to the HA protein of GX/18 virus, were successfully generated. Additionally, four viruses, designated rGX/18, rGD/104, rGX/18-GDHA and rGD/104-GXHA, were rescued to evaluate the cross-reactivity with these antibodies.

As shown in Table 2, the antisera against GX/18 reacted well with $\mathrm{rGX} / 18$ and $\mathrm{rGD} / 104$-GXHA viruses (titers $1: 1,280$ to $1: 2,560$ ) but poorly with rGD/104 and rGX/18-GDHA viruses (titers 1:40 to 1:80). Similarly, the antisera against GD/104 reacted well rGD/104 and rGX/18-GDHA viruses (titers 1:1,280 to $1: 2,560$ ) but poorly with $\mathrm{rGX} / 18$ and $\mathrm{rGD} / 104$-GXHA viruses (titers 1:80 to 1:160). Moreover, the reactive titers of $\mathrm{rGX} / 18$ and $\mathrm{rGD} / 104-\mathrm{GXHA}$ viruses with the mAb102-95 were high up to 1:5,120, which was 32 -fold higher than those of rGD/104 and rGX/18-GDHA viruses with mAb102-95. Since the significantly different reactivity of $\mathrm{rGX} / 18$ and $\mathrm{rGD} / 104$ with mAb102-95, the critical antigenic amino acid was explored by use of mAb102-95 in the following study.

3.3 The amino acid at position 158 in $\mathrm{HA}$ was major determinant for antigenic alteration of EA H1N1 SIVs.

Sequence alignment revealed that the HA1 proteins of GX/18 and GD/104 viruses differed by 25 amino acids 
(Figure 2A). And nine of them have been reported to be located on the antigenic sites Sa (blue), Sb (yellow) and Ca2 (red)(Jin et al., 2005, Tsibane et al., 2012, Koel et al., 2013), as shown in the stimulated 3D structure of GX/18 HA protein generated by using SWISS-MODEL (www. swissmodel.expasy.org) (Waterhouse et al., 2018)and PyMOL software (Figure 2B). To determine whether these nine amino acids contribute to the different antigenicity of GX/18 and GD/104 viruses, we generated four chimeric viruses in the GX/18 background. rGX/18-GDpool contained all the nine different amino acids of GD/104 virus, rGX/18-GDSa contained K156Q, G158E, and K166N mutations in Sa site, rGX/18-GDSb contained T187N, D188Y, S189R, and T193A mutations in Sb site, and rGX/18-GDCa2 contained S138A and N145K mutations in Ca2 site. The cross-reactivity of the four recombinant viruses with the mAb102-95 were evaluated. As shown in Figure 3 , the reactive titers of $\mathrm{rGX} / 18$-GDSb and $\mathrm{rGX} / 18$-GDCa2 viruses with $\mathrm{mAb} 102-95$ were comparable to those of the parental $\mathrm{rGX} / 18$ virus and reached 1:2,560. However, the reactive titers of $\mathrm{rGX} / 18$-GDpool and $\mathrm{rGX} / 18$-GDSa were $8^{\sim} 32$-fold lower than those of the parental $\mathrm{rGX} / 18$ virus and only reached 1:160 and 1:640, respectively. These results demonstrated that the nine different amino acids, especially the Sa antigenic site, played critical roles in viral antigenic drift.

Sa antigenic sites of the GX/18 and GD/104 viruses differed by three amino acids at positions 156, 158, and 166. To further refine the impact of specific mutation on antigenicity, the amino acid substitutions $\mathrm{K} 156 \mathrm{Q}, \mathrm{G} 158 \mathrm{E}$, and $\mathrm{K} 166 \mathrm{~N}$ were individually introduced into the GX/18 virus, and their cross-reactivity with mAb102-95 were assessed by HI tests. As shown in Figure 3, rGX/18-HA-K156Q and rGX/18-HA$\mathrm{K} 166 \mathrm{~N}$ viruses had the similar reactive patterns with the parental $\mathrm{rGX} / 18$ virus, and the HI titers were comparable to the homologous ones. However, the reactive titer of rGX/18-HA-G158E with mAb102-95 was 8-fold lower than the homologous titer and only reached 1:640. Additionally, the E158G mutation substantially increased the cross-reactivity of rGD/104-HA-E158G with the mAb102-95 and the HI titer was up to 1:5,120. These results further indicated that a single amino acid substitution at position 158 in HA protein significantly affected viral antigenicity.

\subsection{The amino acid at position 158 alters virus reactivity with antibodies due to steric effect.}

To investigate how the HA gene affects the antigenic differences between GX/18 and GD/104 viruses, we compared the 3D structure of GX/18-HA-158G and GX/18-HA-158E proteins. As shown in Figure 4, the amino acid mutation from $\mathrm{G}$ to $\mathrm{E}$ caused the $\mathrm{R}$ group changing from $-\mathrm{H}$ to $-\left(\mathrm{CH}_{2}\right)_{2}-\mathrm{COOH}$, and the consequent steric effect directly hindered the recognition of antibody to HA protein. Additionally, the polarity of $-\mathrm{H}$ was almost negligible compared to that of $-\left(\mathrm{CH}_{2}\right)_{2}-\mathrm{COOH}$, so E was comparatively more hydrophilic than G. Since the hydrophobic interaction is the principle driving force for protein folding(Zhu et al., 2016a), the G158E mutation might also affect the local folding of HA protein through altering the degrees of hydrophobicity and further impact the binding affinity with antibody, resulting in antigenic drift ultimately.

\section{Discussion}

EA H1N1 SIVs have been circulating in pig herbs in Europe and Asia for decades. Continual mutations and recombination with other viruses have entitled EA H1N1 SIVs to form distinct antigenic groups, with markedly different antigenicity from those of the pdm/09 H1N1 viruses. And antigenic variants escaping pre-existing immunity might cause outbreaks in pigs and pose greater risks to human health. In this study, we used A/swine/Guangxi/18/2011 and A/swine/Guangdong/104/2013 as models to explore the genetic basis of antigenic drift of EA H1N1 SIVs, and found that a single amino acid at position 158 in Sa antigenic site substantially contributed to the antigenic variation.

Five major antigenic sites, $\mathrm{Sa}, \mathrm{Sb}, \mathrm{Ca} 1, \mathrm{Ca} 2$ and $\mathrm{Cb}$, have been mapped onto the HA1 protein and antibodies targeting each of which can neutralize the infectivity of influenza virus. The immunodominant site Sa is located proximal to the receptor binding site and elicits high potency neutralizing antibodies(Shembekar et al., 2013). In the past decades, amino acid mutations in Sa site have induced antigenic variability of different subtype influenza viruses. The pdm/09 H1N1 continues to circulate worldwide and cause periodic outbreaks in human population. Boivin et al . previously reported that A/California/07/09 vaccine cannot protect mice 
against lethal infection of rescued virus with G158E and N159D mutations in the Sa antigenic site(Retamal et al., 2017). Smith et al . created an antigenic evolution map using HI assay data and demonstrated that amino acid changes in Sa site repeatedly involved in antigenic variations of H3N2 influenza viruses(Smith et al., 2004). In the present study, G158E mutation considerably decreased the reactivity of GX/18 virus with the homologous antibodies, further suggesting Sa antigenic site played a pivotal role in antigenic drift of EA H1N1 SIVs.

Amino acid mutations can affect influenza virus antigenicity in a variety of ways, such as altering glycosylation of HA protein and the binding affinity between epitope and antibody. Previous study demonstrated that E131N mutation changed the antigenic properties of H5N1 avian influenza viruses through forming a new N-linked glycosylation site at positions 131-133(Gu et al., 2019). Here, we found that G158E substitution contributed to the antigenic drift of EA H1N1 SIVs via decreasing the binding affinity between HA and antibody. Accompanied with G158E mutation, the R group changed from - $\mathrm{H}$ to $-\left(\mathrm{CH}_{2}\right)_{2}$ - $\mathrm{COOH}$, resulting in the alteration of spatial structure and hydrophobicity. The umbrella-like sidechain of $\mathrm{E}$ and its improved hydrophilicity might jointly block the interaction between HA protein and antibody.

Notably, G158E mutation substantially decreased, rather than eliminated, the reactivity of GX/18 with mAb102-95, suggesting other amino acid(s) acts as assistant to unitedly cause antigenic variation. Moreover, Sunet al . identified $77 \mathrm{EA} \mathrm{H1N1}$ SIVs, isolated in slaughterhouse in 10 provinces in China from 2011 to 2018, and found these viruses could be classified into two antigenic groups based on the HI assays(Sun et al., 2020). However, sequence analysis showed that $158 \mathrm{G}$, not $158 \mathrm{E}$, were conserved in all the 77 viruses (data not shown), indicating that the $158 \mathrm{G}$-related viruses are still the predominant strains in China currently. The underlying mechanism of G158E altering the antigenic property of EA H1N1 SIVs remains ambiguous and the other amino acids behind antigenic drift still needs to be further investigated. These data revealed that EA H1N1 SIVs have been evolving in a complex direction and highlight the importance of active surveillance and evaluating the virus antigenic properties.

\section{Acknowledgements}

This work was supported by the grants from Natural Science Foundation of Henan Province (Grant No. 202300410198) and National Natural Science Foundation of China (Grant No. 32002259).

\section{Conflict of interest}

The authors declared no potential conflict of interests with respect to the research, authorship, and publication of this article.

\section{References}

Cao, Z. P., W. J. Zeng, X. Q. Hao, J. M. Huang, M. K. Cai, P. Zhou and G. H. Zhang, 2019: Continuous evolution of influenza A viruses of swine from 2013 to 2015 in Guangdong, China.PLoS One, 14.

Chi, X. S., T. V. Bolar, P. Zhao, J. S. Tam, R. Rappaport and S. M. Cheng, 2005: Molecular evolution of human influenza A/H3N2 virus in Asia and Europe from 2001 to 2003. J. Clin. Microbiol., 43, 6130-6132.

Gefter, M. L., D. H. Margulies and M. D. Scharff, 1977: A simple method for polyethylene glycol-promoted hybridization of mouse myeloma cells. Somatic Cell Genet., 3, 231-236.

Gu, C., X. Zeng, Y. Song, Y. Li, L. Liu, Y. Kawaoka, D. Zhao and H. Chen, 2019: Glycosylation and an amino acid insertion in the head of hemagglutinin independently affect the antigenic properties of H5N1 avian influenza viruses. Science China. Life sciences, 62, 76-83.

He, P., G. J. Wang, Y. N. Mo, Q. X. Yu, X. Xiao, W. J. Yang, W. F. Zhao, X. Guo, Q. Chen, J. Q. He, M. L. Liang, J. Zhu, Y. B. Ding, Z. Z. Wei, K. Ouyang, F. Liu, H. Jian, W. J. Huang, A. Garcia-Sastre and Y. Chen, 2018: Novel triple-reassortant influenza viruses in pigs, Guangxi, China. Emerging microbes Es infections, 7. 
Ito, T., J. N. Couceiro, S. Kelm, L. G. Baum, S. Krauss, M. R. Castrucci, I. Donatelli, H. Kida, J. C. Paulson, R. G. Webster and Y. Kawaoka, 1998: Molecular basis for the generation in pigs of influenza A viruses with pandemic potential.J. Virol., 72, 7367-7373.

Jin, H., H. Zhou, H. Liu, W. Chan, L. Adhikary, K. Mahmood, M. S. Lee and G. Kemble, 2005: Two residues in the hemagglutinin of A/Fujian/411/02-like influenza viruses are responsible for antigenic drift from A/Panama/2007/99. Virology, 336, 113-119.

Koel, B. F., D. F. Burke, T. M. Bestebroer, S. van der Vliet, G. C. Zondag, G. Vervaet, E. Skepner, N. S. Lewis, M. I. Spronken, C. A. Russell, M. Y. Eropkin, A. C. Hurt, I. G. Barr, J. C. de Jong, G. F. Rimmelzwaan, A. D. Osterhaus, R. A. Fouchier and D. J. Smith, 2013: Substitutions near the receptor binding site determine major antigenic change during influenza virus evolution.Science, 342, 976-979.

Li, Y., Y. Li, M. Ye, D. Wang, J. Zhao, X. Sun, Q. Mao and H. Xia, 2017: Biological function analysis of monoclonal antibodies against human granulins in vitro using U251 cells as a model. Protein Expr. Purif., $130,55-62$.

Ma, W., R. E. Kahn and J. A. Richt, 2008: The pig as a mixing vessel for influenza viruses: Human and veterinary implications. J. Mol. Genet. Med., 3, 158-166.

Pensaert, M., K. Ottis, J. Vandeputte, M. M. Kaplan and P. A. Bachmann, 1981: Evidence for the natural transmission of influenza A virus from wild ducts to swine and its potential importance for man. Bull. World Health Organ., 59, 75-78.

Pereda, A., J. Cappuccio, M. A. Quiroga, E. Baumeister, L. Insarralde, M. Ibar, R. Sanguinetti, M. L. Cannilla, D. Franzese, O. E. Escobar Cabrera, M. I. Craig, A. Rimondi, M. Machuca, R. T. Debenedetti, C. Zenobi, L. Barral, R. Balzano, S. Capalbo, A. Risso and C. J. Perfumo, 2010: Pandemic (H1N1) 2009 outbreak on pig farm, Argentina. Emerg. Infect. Dis., 16, 304-307.

Ping, J., C. Li, G. Deng, Y. Jiang, G. Tian, S. Zhang, Z. Bu and H. Chen, 2008: Single-amino-acid mutation in the HA alters the recognition of H9N2 influenza virus by a monoclonal antibody. Biochem. Biophys. Res. Commun., 371, 168-171.

Retamal, M., Y. Abed, C. Rheaume, M. Baz and G. Boivin, 2017: In vitro and in vivo evidence of a potential A(H1N1)pdm09 antigenic drift mediated by escape mutations in the haemagglutinin Sa antigenic site. $J$. Gen. Virol., 98, 1224-1231.

Shembekar, N., V. V. A. Mallajosyula, A. Mishra, L. Yeolekar, R. Dhere, S. Kapre, R. Varadarajan and S. K. Gupta, 2013: Isolation of a High Affinity Neutralizing Monoclonal Antibody against 2009 Pandemic H1N1 Virus That Binds at the 'Sa' Antigenic Site. PLoS One, 8.

Smith, D. J., A. S. Lapedes, J. C. de Jong, T. M. Bestebroer, G. F. Rimmelzwaan, A. D. M. E. Osterhaus and R. A. M. Fouchier, 2004: Mapping the antigenic and genetic evolution of influenza virus. Science, 305, $371-376$.

Smith, D. J., A. S. Lapedes, S. Forrest, J. C. de Jong, A. D. M. E. Osterhaus, R. A. M. Fouchier, N. J. Cox and A. S. Perelson, 2001: Modeling the effects of updating the influenza vaccine on the efficacy of repeated vaccination. Options for the Control of Influenza Iv, 1219, 655-660.

Sun, H., Y. Xiao, J. Liu, D. Wang, F. Li, C. Wang, C. Li, J. Zhu, J. Song, H. Sun, Z. Jiang, L. Liu, X. Zhang, K. Wei, D. Hou, J. Pu, Y. Sun, Q. Tong, Y. Bi, K. C. Chang, S. Liu, G. F. Gao and J. Liu, 2020: Prevalent Eurasian avian-like H1N1 swine influenza virus with 2009 pandemic viral genes facilitating human infection. Proc. Natl. Acad. Sci. U. S. A., 117, 17204-17210.

Tsibane, T., D. C. Ekiert, J. C. Krause, O. Martinez, J. E. Crowe, Jr., I. A. Wilson and C. F. Basler, 2012: Influenza human monoclonal antibody $1 \mathrm{~F} 1$ interacts with three major antigenic sites and residues mediating human receptor specificity in H1N1 viruses. PLoS Pathog., 8, e1003067. 
Wang, Z., H. Yang, Y. Chen, S. Tao, L. Liu, H. Kong, S. Ma, F. Meng, Y. Suzuki, C. Qiao and H. Chen, 2017: A Single-Amino-Acid Substitution at Position 225 in Hemagglutinin Alters the Transmissibility of Eurasian Avian-Like H1N1 Swine Influenza Virus in Guinea Pigs. J. Virol., 91.

Waterhouse, A., M. Bertoni, S. Bienert, G. Studer, G. Tauriello, R. Gumienny, F. T. Heer, T. A. P. de Beer, C. Rempfer, L. Bordoli, R. Lepore and T. Schwede, 2018: SWISS-MODEL: homology modelling of protein structures and complexes.Nucleic Acids Res., 46, W296-W303.

Weingartl, H. M., Y. Berhane, T. Hisanaga, J. Neufeld, H. Kehler, C. Emburry-Hyatt, K. Hooper-McGreevy, S. Kasloff, B. Dalman, J. Bystrom, S. Alexandersen, Y. Li and J. Pasick, 2010: Genetic and Pathobiologic Characterization of Pandemic H1N1 2009 Influenza Viruses from a Naturally Infected Swine Herd. J. Virol., $84,2245-2256$.

Xie, J. F., Y. H. Zhang, L. Zhao, W. Q. Xiu, H. B. Chen, Q. Lin, Y. W. Weng and K. C. Zheng, 2018: Emergence of Eurasian Avian-Like Swine Influenza A (H1N1) Virus from an Adult Case in Fujian Province, China. Virol. Sin., 33, 282-286.

Yang, H. L., Y. Chen, C. L. Qiao, X. J. He, H. Zhou, Y. Sun, H. Yin, S. S. Meng, L. P. Liu, Q. Y. Zhang, H. H. Kong, C. Y. Gu, C. J. Li, Z. G. Bu, Y. Kawaoka and H. L. Chen, 2016: Prevalence, genetics, and transmissibility in ferrets of Eurasian avian-like H1N1 swine influenza viruses. Proc. Natl. Acad. Sci. U. S. A., 113, 392-397.

Yang, H. L., C. L. Qiao, X. Tang, Y. Chen, X. G. Xin and H. L. Chen, 2012: Human Infection from Avian-like Influenza A (H1N1) Viruses in Pigs, China. Emerging Infect. Dis.,18, 1144-1146.

Zhu, C. Q., Y. R. Gao, H. Li, S. Meng, L. Li, J. S. Francisco and X. C. Zeng, 2016a: Characterizing hydrophobicity of amino acid side chains in a protein environment via measuring contact angle of a water nanodroplet on planar peptide network. Proc. Natl. Acad. Sci. U. S. A., 113, 12946-12951.

Zhu, H. C., B. P. Zhou, X. H. Fan, T. T. Y. Lam, J. Wang, A. Chen, X. C. Chen, H. L. Chen, R. G. Webster, R. Webby, J. S. M. Peiris, D. K. Smith and Y. Guan, 2011: Novel Reassortment of Eurasian Avian-Like and Pandemic/2009 Influenza Viruses in Swine: Infectious Potential for Humans. J. Virol., 85, 10432-10439.

Zhu, H. N., B. Damdinjav, G. Gonzalez, L. V. Patrono, H. Ramirez-Mendoza, J. A. R. Amat, J. Crispell, Y. A. Parr, T. A. Hammond, E. Shiilegdamba, Y. H. C. Leung, M. Peiris, J. F. Marshall, J. Hughes, M. Gilbert and P. R. Murcia, 2019a: Absence of adaptive evolution is the main barrier against influenza emergence in horses in Asia despite frequent virus interspecies transmission from wild birds. PLoS Path., 15.

Zhu, W. F., Z. M. Feng, Y. K. Chen, L. Yang, J. Liu, X. Y. Li, S. L. Liu, L. J. Zhou, H. J. Wei, R. B. Gao, D. Y. Wang and Y. L. Shu, 2019b: Mammalian-adaptive mutation NP-Q357K in Eurasian H1N1 Swine Influenza viruses determines the virulence phenotype in mice. Emerging microbes $\&$ infections, 8, 989-999.

Zhu, W. F., H. Zhang, X. Y. Xiang, L. L. Zhong, L. Yang, J. F. Guo, Y. R. Xie, F. C. Li, Z. H. Deng, H. Feng, Y. W. Huang, S. X. Hu, X. Xu, X. H. Zou, X. D. Li, T. Bai, Y. K. Chen, Z. Li, J. H. Li and Y. L. Shu, 2016b: Reassortant Eurasian Avian-Like Influenza A(H1N1) Virus from a Severely Ill Child, Hunan Province, China, 2015. Emerging Infect. Dis., 22, 1930-1936.

\section{Figure Legends}

Figure 1 Antigenic cartography of EA H1N1 SIVs. Filled squares and circles represent the positions of antisera and viruses, respectively. The spacing of one gridline corresponds to an HI measurement, which equals a 2-fold difference in the HI assay. All the three axes represent antigenic distance. Strains belonging to the same antigenic cluster are encircled in a circle. Details of the HI assay data have been reported previously(Yang et al., 2016).

Figure 2 (A) Amino acid differences in HA1 protein between GX/18 and GD/104 viruses are shown as single letters at the indicated positions. Each amino acid of GX/18 is shown before the number of the position, and that of GD/104 is shown after the number of the position. (B) 3D structure of the HA protein with 9 
different amino acids. The 3D structure of GX/18-HA protein is predicted by SWISS-MODEL and analyzed with PyMOL software. Different amino acids in HA1 protein are marked with different color. Residues located in antigenic site Sa are labeled as blue, site $\mathrm{Sb}$ as yellow, and site $\mathrm{Ca} 2$ as red. ${ }^{*}$, since the amino acid at position 175 is buried internally, the effect of E175R mutation on antigenicity was not evaluated.

Figure 3 The reactivity of rGX/18 viruses bearing different amino acid mutations in HA with mAb102-95. The reactivity was tested by using the HI assay and the dashed line indicates the limit of detection.

Figure 4 Structural analysis of amino acid mutation G158E. The structural change from 158G to $158 \mathrm{E}$ is analyzed by use of PyMOL software, and the figure only shows the difference of $\mathrm{R}$ group.

TABLE 1 Primers used for pBD cDNA construction and for introducing mutations into the HA gene of the reassortant and mutant viruses.

\begin{tabular}{lll}
\hline & Primers $\left(5^{\prime}-3^{\prime}\right)^{a}$ & Primers $\left(5^{\prime}-3^{\prime}\right)^{a}$ \\
\hline Purpose & Forward & Reverse \\
PB2 amplification & CCAGCAAAAGCAGGTCAAATATATTCAA & TTAGTAGAAACAAGGTCGTT1 \\
PB1 amplification & CCAGCAAAAGCAGGCAAACCATTTGA & TTAGTAGAAACAAAGGCATTT \\
HA amplification & CCAGCAAAAGCAGGTACTGAT & TTAGTAGAAACAAGGTACTTT \\
NP amplification & CCAGCAAAAGCAGGGGAAAATT & TTAGTAGAAACAAGGGTGTT \\
NA amplification & CCAGCAAAAGCAGGGTAGATAATCACTCA & TTAGTAGAAACAAGGGTATTT \\
M amplification & CCAGCAAAAGCAGGAGTTTAAAATG & TTAGTAGAAACAAGGAGTTT \\
NS amplification & CCAGCAAAAGCAGGTAGATAT & TTAGTAGAAACAAGGTAGTT \\
GX/18-HA1-S138A & CCAGCAAAAGCAGGGTGACAAA & TTAGTAGAAACAAGGGTGTT \\
GX/18-HA1-N145K & GTACCACAGTTGCATGCTCCCAC & CAACTGTGGTACCTCTGGTG \\
GX/18-HA1-K156Q & CACTCTGGAGCCAAAAGCTTTTATC & TTTGGCTCCAGAGTGGGAGC \\
GX/18-HA1-G158E & TTTACTATGGATAGTACAGAAAGGAAACT & GTACTATCCATAGTAAATTCC \\
GX/18-HA1-K166N & ATGGATAGTAAAGAAAGAAACTCC & TCTTTCTTTACTATCCATAGT \\
GX/18-HA1-T193A & ATCCTAAGCTCAGCAATTCATACAC & ATTGCTGAGCTTAGGATAGGA \\
GX/18-HA1-T187N/D188Y/S189R & CAGAGACCAACAAGCCCTCTACCAG & CTTGTTGGTCTCTGTAGTTC \\
\hline
\end{tabular}

${ }^{a}$ Nucleotides that were changed are underlined and in boldface type.

TABLE 2 Antigenic analysis of the EA H1N1 SIVs

\begin{tabular}{llllll}
\hline Virus & Ferret antiserum $^{a}$ & Ferret antiserum $^{a}$ & Guinea pig antiserum & & Guinea pig antiserum \\
& & $\mathrm{mAb}^{b}$ & G $^{b}$ \\
rGX/18 & GX/18 & GD/104 & GX/18 & GD/104 & 102-95 \\
rGD/104 & 2560 & 80 & 2560 & 160 & 5120 \\
rGX/18-GDHA & 80 & 2560 & 40 & 1280 & 160 \\
rGD/104-GXHA & 1280 & 1280 & 40 & 1280 & 160 \\
\hline
\end{tabular}

${ }^{a}$ Homologous titers are underlined and bolded.

${ }^{b} \mathrm{mAb}$, monoclonal antibody

\section{Hosted file}

Figures.pptx available at https://authorea.com/users/415835/articles/523610-a-single-aminoacid-at-position-158-in-hemagglutinin-affects-the-antigenic-property-of-eurasian-avianlike-h1n1-swine-influenza-viruses 